\title{
ADAPTIVE STABILIZATION OF UNSTABLE STEADY STATES OF NONLINEAR DYNAMICAL SYSTEMS*
}

\author{
V. Pyragas and K. Pyragas \\ Semiconductor Physics Institute, A. Goštauto 11, LT-01108 Vilnius, Lithuania \\ E-mail: viktpy@pfi.lt,pyragas@kes0.pfi.lt
}

Received 2 October 2003

\begin{abstract}
An adaptive dynamic state feedback controller for stabilizing and tracking unknown steady states of dynamical systems is considered. We prove that the steady state can never be stabilized if the system and controller as a whole has an odd number of real positive eigenvalues. For two-dimensional systems, this topological limitation states that only an unstable focus or node can be stabilized with a stable controller, and a stabilization of a saddle requires the presence of an unstable degree of freedom in a feedback loop. The use of the controller to stabilize saddles and unstable spirals is demonstrated numerically with several models: a pendulum driven with a constant torque, the Lorenz system, and an electrochemical Ni dissolution system.
\end{abstract}

Keywords: chaos, dynamical systems, adaptive control, electrochemical oscillator, basin of attraction

PACS: $05.45 . \mathrm{Gg}, 02.30 . \mathrm{Yy}, 02.30 . \mathrm{Ks}$

\section{Introduction}

The control theory is one of the central subjects in engineering science. It has been studied for at least half a century and has profited from a huge formal apparatus developed in applied mathematics [1,2]. In fact, the scope of the general control theory is rather wide and covers almost all possible perturbations of dynamical systems, which intend to direct their motion into a desired state.

Within this background one might wonder why physicists have revived their interest to the field of control in the last decade, with an emphasis on nonlinear and especially chaotic dynamical systems. The interest came from the observation that chaotic dynamical systems admit a huge number of unstable periodic orbits. Since these orbits represent genuine motions of the system under consideration one can expect to achieve stabilization by applying tiny control forces. Hence, chaotic dynamics opens the possibility to control quite distinct types of motion with a low control power.

The idea of controlling chaos has been first formulated by Ott et al. in 1990 [3]. It stimulated development of rich variety of new control techniques (see [4] for a review), among which the delayed feedback control method [5] has gained widespread accep-

\footnotetext{
* The report presented at the 35th Lithuanian National Physics Conference, 12-14 June 2003, Vilnius, Lithuania.
}

tance. This method provides the stabilization of a desired orbit without knowledge of the system equations. It has been successfully implemented in various experimental systems. However, Nakajima [6] proved a topological limitation that the method cannot stabilize the torsion-free periodic orbits, which are characterized by an odd number of real positive Floquet exponents. Recently, it has been shown that an additional unstable degree of freedom introduced into a feedback loop can overcome this limitation [7].

Although the field of controlling chaos deals mainly with stabilization of unstable periodic orbits, the problem of stabilizing unstable steady states of dynamical systems is of great importance for various technical applications. Stabilization of a fixed point by usual methods of classical control theory requires a knowledge of its location in the phase space. However, for many complex systems (e.g., chemical or biological), the location of the fixed points, as well as exact model equations, are unknown. In this case, adaptive control techniques capable of automatically locating the unknown steady states are preferable. An adaptive stabilization of a fixed point can be attained with the delayed feedback method $[5,8,9]$. However, the use of the time-delayed signals in this problem is not necessary, and thus the difficulties related to the infinitedimensional phase space due to delay can be avoided. A simpler adaptive controller for stabilizing unknown 
steady states can be designed on the basis of ordinary differential equations (ODEs). The simplest example of such a controller utilizes a conventional low-pass filter described by one ODE. The filtered dc output signal of the system estimates the location of the fixed point, so that the difference between the actual and the filtered output signals can be used as a control signal. Efficiency of such a simple controller has been demonstrated for different experimental systems [9-11].

In this paper we consider a generalized adaptive controller described by a system of ODEs and prove that it has a topological limitation concerning an odd number of real positive eigenvalues of the steady state. We show that the limitation can be overcome by implementing an unstable degree of freedom into a feedback loop. The efficiency of the adaptive controller is demonstrated with several models, namely, the Lorenz system, a pendulum driven by a constant torque, and electrochemical dissolution of nickel in sulphuric acid.

\section{Adaptive controller}

\subsection{Controller based on the low-pass filter}

An adaptive controller based on the conventional low-pass filter, which was successfully used in several experiments [9-11], is not universal. This can be illustrated with a simple example. Consider a onedimensional dynamical system $\dot{x}=\lambda^{\mathrm{s}}\left(x-x^{*}\right)$ having a single unstable steady state $x=x^{*}$ with the eigenvalue $\lambda^{\mathrm{s}}>0$. We assume that the location of the fixed point $x^{*}$ is unknown and test a possibility of its stabilization by means of the dynamic controller based on a low-pass filter,

$$
\begin{aligned}
& \dot{x}=\lambda^{\mathrm{s}}\left(x-x^{*}\right)+k(w-x), \\
& \dot{w}=\lambda^{\mathrm{c}}(w-x) .
\end{aligned}
$$

Here $w$ is a dynamic variable of the controller. The equation $\dot{w}=\lambda^{\mathrm{c}}(w-x)$ for $\lambda^{\mathrm{c}}<0$ represents a conventional low-pass filter, rc circuit, with the time constant $\tau=-1 / \lambda^{\mathrm{c}}$. The control signal $k(w-x)$ is added as a feedback to the controlled system $\dot{x}=\lambda^{\mathrm{s}}\left(x-x^{*}\right)$. Note that the controller is designed in such a way that it does not change the location of the fixed point of the free system. The fixed point of the closed-loop system in the whole phase space of variables $(x, w)$ is $\left(x^{*}, x^{*}\right)$ so that its projection onto the $x$ axis corresponds to the fixed point of the free system at arbitrary control gain $k$. If for some values of $k$ the closed-loop system is stable, the controller variable $w$ converges to the steady-state value $w^{*}=x^{*}$ and the feedback perturbation vanishes.
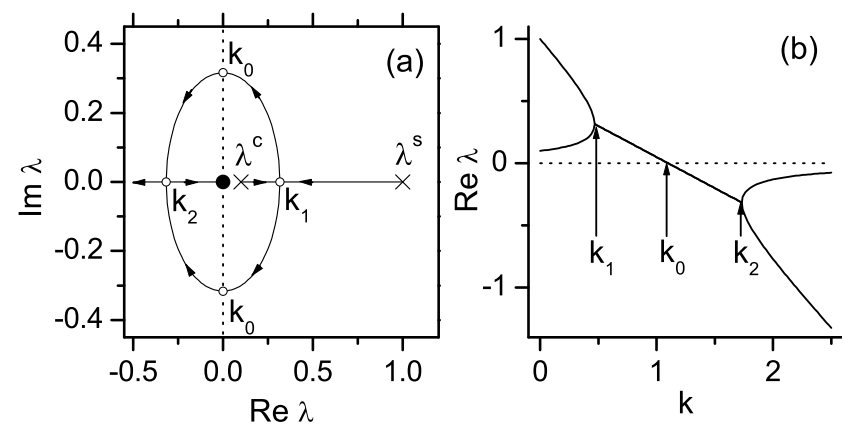

Fig. 1. Stabilization of an unstable fixed point with an unstable controller in a simple model of Eq. (1) for $\lambda^{\mathrm{s}}=1$ and $\lambda^{\mathrm{c}}=0.1$. (a) Root loci of the characteristic equation as $k$ varies from 0 to $\infty$. The crosses and solid dot denote the root locations at $k=0$ and $k \rightarrow \infty$, respectively. (b) $\operatorname{Re} \lambda$ versus $k$. Here $k_{0}=\lambda^{\mathrm{s}}+\lambda^{\mathrm{c}}$ and $k_{1,2}=\lambda^{\mathrm{s}}+\lambda^{\mathrm{c}} \mp 2 \sqrt{\lambda^{\mathrm{s}} \lambda^{\mathrm{c}}}$.

The closed-loop system is stable if both eigenvalues of the characteristic equation $\lambda^{2}-\left(\lambda^{\mathrm{s}}+\lambda^{\mathrm{c}}-k\right) \lambda+$ $\lambda^{\mathrm{s}} \lambda^{\mathrm{c}}=0$ are in the left half-plane, $\operatorname{Re} \lambda<0$. The stability conditions are $k>\lambda^{\mathrm{s}}+\lambda^{\mathrm{c}}, \lambda^{\mathrm{s}} \lambda^{\mathrm{c}}>0$. We see immediately that the stabilization is not possible with a conventional low-pass filter, since for any $\lambda^{\mathrm{s}}>0$ and $\lambda^{\mathrm{c}}<0$ we have $\lambda^{\mathrm{s}} \lambda^{\mathrm{c}}<0$, and the second stability condition is not satisfied. However, the stabilization can be attained via an unstable controller with a positive parameter $\lambda^{\mathrm{c}}$. Electronically, such a controller can be implemented as the rc circuit with a negative resistance. Figure 1 shows the mechanism of the stabilization. For $k=0$, the eigenvalues are $\lambda^{\mathrm{s}}$ and $\lambda^{\mathrm{c}}$, which correspond to the free system and the free controller, respectively. With the increase of $k$, they approach each other on the real axis, then collide at $k=k_{1}$, and pass to the complex plane. At $k=k_{0}$ they cross symmetrically into the left half-plane (Hopf bifurcation). At $k=k_{2}$ there is another collision on the real axis, after which one of the roots moves towards $-\infty$ while the second one approaches the $\lambda$-plane origin. For $k>k_{0}$, the closedloop system is stable. An optimal value of the control gain is $k_{2}$, since it provides the fastest convergence to the fixed point.

\subsection{Generalized adaptive controller}

Now we consider the problem of adaptive stabilization in general. Let

$$
\dot{\mathbf{x}}=\mathbf{f}(\mathbf{x}, \mathbf{p})
$$

be the dynamical system with the $N$-dimensional vector variable $\mathrm{x}$ and and the $L$-dimensional vector parameter $\mathbf{p}$ available for an external adjustment. Assume that the $n$-dimensional vector variable $\mathbf{y}(t)=\mathbf{g}(\mathbf{x}(t))$, 
which is a function of the dynamical variables $\mathbf{x}(t)$, represents the system output. Suppose that at $\mathbf{p}=\mathbf{p}_{0}$ the system has an unstable fixed point $\mathrm{x}^{*}$ that satisfies $\mathbf{f}\left(\mathbf{x}^{*}, \mathbf{p}_{0}\right)=0$. The location of the fixed point $\mathbf{x}^{*}$ is unknown. To stabilize the fixed point we perturb the parameters by an adaptive feedback

$$
\mathbf{p}(t)=\mathbf{p}_{0}+k B[A \mathbf{w}(t)+C \mathbf{y}(t)],
$$

where $\mathbf{w}$ is the $M$-dimensional dynamical variable of the controller that satisfies the equation

$$
\dot{\mathbf{w}}(t)=A \mathbf{w}+C \mathbf{y} .
$$

Here $A, B$, and $C$ are the matrices of dimensions $M \times M, M \times L$, and $n \times M$, respectively, and $k$ is a scalar parameter that defines the feedback gain. The feedback is constructed in such a way that it does not change the steady-state solutions of the free system. For any $k$, the fixed point of the closed-loop system is $\left\{\mathbf{x}^{*}, \mathbf{w}^{*}\right\}$, where $\{\mathbf{x}, \mathbf{w}\}$ denotes the phase space of variables, $\mathrm{x}^{*}$ is the fixed point of the free system, and $\mathbf{w}^{*}$ is a corresponding steady-state value of the controller variable. The latter satisfies the system of linear equations $A \mathbf{w}^{*}=-C \mathbf{g}\left(\mathrm{x}^{*}\right)$ which has a unique solution for any nonsingular matrix $A$. The feedback perturbation $k B \dot{\mathbf{w}}$ vanishes whenever the fixed point of the closed-loop system is stabilized.

Small deviations $\delta \mathbf{x}=\mathbf{x}-\mathbf{x}^{*}$ and $\delta \mathbf{w}=\mathbf{w}-\mathbf{w}^{*}$ from the fixed point are described by the variational equations

$$
\begin{aligned}
\delta \dot{\mathbf{x}} & =J \delta \mathbf{x}+k P B \delta \dot{\mathbf{w}}, \\
\delta \dot{\mathbf{w}} & =C G \delta \mathbf{x}+A \delta \mathbf{w},
\end{aligned}
$$

where $J=D_{\mathbf{x}} \mathbf{f}\left(\mathbf{x}^{*}, \mathbf{p}_{0}\right), P=D_{\mathbf{p}} \mathbf{f}\left(\mathbf{x}^{*}, \mathbf{p}_{0}\right)$, and $G=D_{\mathrm{x}} \mathbf{g}\left(\mathrm{x}^{*}\right)$. Here $D_{\mathrm{x}}$ and $D_{\mathbf{p}}$ denote the vector derivatives (Jacobian matrices) with respect to the variables $\mathbf{x}$ and parameters $\mathbf{p}$, respectively. The characteristic equation for the closed-loop system has the form

$$
\Delta_{k}(\lambda) \equiv\left|\begin{array}{cc}
I \lambda-J & -k \lambda P B \\
-C G & I \lambda-A
\end{array}\right|=0 .
$$

For $k=0$, we have $\Delta_{0}(\lambda)=|I \lambda-J||I \lambda-A|$ and Eq. (6) splits into two independent equations $|I \lambda-J|=0$ and $|I \lambda-A|=0$ that define $N$ eigenvalues of the free system, $\lambda=\lambda_{j}^{\mathrm{s}}, j=1, \ldots, N$, and $M$ eigenvalues of the free controller, $\lambda=\lambda_{j}^{\mathrm{c}}$, $j=1, \ldots, M$, respectively. By assumption, at least one eigenvalue of the free system is in the right halfplane. The closed-loop system is stabilized in an interval of the control gain $k$ for which all eigenvalues of Eq. (6) are in the left half-plane, $\operatorname{Re} \lambda<0$.

\subsection{Limitation of the generalized controller}

Now we prove an important topological limitation of the above-described adaptive controller. It is similar to the Nakajima theorem [6] concerning the limitation of the time-delayed feedback controller.

Consider a fixed point $\mathrm{x}^{*}$ of the dynamical system (2) characterized by Jacobian matrix $J$ and the adaptive controller (4) with a nonsingular matrix $A$. We assert that if the total number of real positive eigenvalues of the matrices $J$ and $A$ is odd, then the closed-loop system described by Eqs. (2)-(4) cannot be stabilized by any choice of matrices $A, B, C$ and control gain $k$.

The proof of the statement is based on the analysis of roots of $\Delta_{k}(\lambda)$ which define the stability of the closedloop system. Rewriting Eq. (6) for $k=0$ in the basis where matrices $J$ and $A$ are diagonal, one obtains

$$
\Delta_{0}(\lambda)=\prod_{j=1}^{N}\left(\lambda-\lambda_{j}^{\mathrm{s}}\right) \prod_{m=1}^{M}\left(\lambda-\lambda_{m}^{\mathrm{c}}\right) .
$$

Here $\lambda_{j}^{\mathrm{s}}$ and $\lambda_{m}^{\mathrm{c}}$ are the eigenvalues of the matrices $J$ and $A$, respectively. Now from Eq. (6) we also have the relation $\Delta_{k}(0)=\Delta_{0}(0)$ and, therefore, Eq. (7) implies

$$
\Delta_{k}(0)=\prod_{j=1}^{N}\left(-\lambda_{j}^{\mathrm{s}}\right) \prod_{m=1}^{M}\left(-\lambda_{m}^{\mathrm{c}}\right)
$$

for all $k$. Since the total number of real and positive eigenvalues $\lambda_{j}^{\mathrm{s}}$ and $\lambda_{m}^{\mathrm{c}}$ is odd and other eigenvalues are either real and negative or come in complex conjugate pairs, $\Delta_{k}(0)$ must be real and negative. On the other hand, from the definition of $\Delta_{k}(\lambda)$ we see immediately that $\lim _{\lambda \rightarrow \infty} \Delta_{k}(\lambda) \rightarrow \lambda^{N+M}>0$ for all $k$. Since the polynomial $\Delta_{k}(\lambda)$ is continuous for all $\lambda$ and it is negative for $\lambda=0$ and is positive for large $\lambda$, it follows that $\Delta_{k}(\lambda)=0$ for some real positive $\lambda$. Thus the closed-loop system always has at least one real positive eigenvalue and cannot be stabilized.

This limitation can be explained by bifurcation theory, similarly to [6]. If a fixed point with an odd total number of real positive eigenvalues is stabilized, one of such eigenvalues must cross into the left half-plane on the real axis accompanied by coalescence of fixed points. However, this contradicts the fact that the feedback perturbation does not change locations of fixed points.

From this limitation it follows that any fixed point $\mathrm{x}^{*}$ with an odd number of real positive eigenvalues cannot be stabilized with a stable controller. In other words, if the Jacobian $J$ of a fixed point has an odd number of real positive eigenvalues then it can be stabilized only 
with an unstable controller, matrix $A$ of which has an odd number (at least one) of real positive eigenvalues.

In the rest of the paper we illustrate the efficiency of an adaptive unstable controller for several physical models.

\section{Control of a pendulum driven by a constant torque}

Consider a simple mechanical example of a nonlinear oscillator: a pendulum driven by a constant torque. The equation of motion in dimensionless form reads

$$
\ddot{\Theta}+\beta \dot{\Theta}+\sin \Theta=\gamma .
$$

Here $\Theta$ denotes the angle between the pendulum and the downward vertical (see inset in Fig. 2), $\beta=$ $b /\left(m L^{3 / 2} g^{1 / 2}\right)$ and $\gamma=\Gamma /(m g L)$ are the dimensionless parameters, $m$ is the mass and $L$ is the length of the pendulum, $b$ is the viscous damping constant, $g$ is the free fall acceleration, and $\Gamma$ is the applied constant torque. The time variable is normalized to the period $T=(L / g)^{1 / 2}$ of the free pendulum oscillations.

For a small torque $\gamma<1$, the pendulum has two equilibrium steady states, coordinates of which in the $(\Theta, \dot{\Theta})$ phase plane are $\left(\Theta_{\mathrm{s}}, 0\right)$ and $\left(\Theta_{\mathrm{u}}, 0\right)$, where $\Theta_{\mathrm{s}}=\arcsin \gamma$ and $\Theta_{\mathrm{u}}=\pi-\arcsin \gamma$. In these steady states the gravity is balanced by the applied torque. The first state corresponds to a stable node or spiral while the second one is a saddle point. Our goal is to stabilize the saddle point $\left(\Theta_{\mathrm{u}}, 0\right)$ by using an unstable adaptive controller based on the rc circuit with a negative resistance.

We suppose that an observable is the angle $\Theta$ and that we can control the system by applying a feedback perturbation to the torque $\gamma$. Then our controlled system is

$$
\begin{aligned}
& \dot{\Theta}=\Omega, \\
& \dot{\Omega}=-\beta \Omega-\sin \Theta+\gamma+k(w-\Theta), \\
& \dot{w}=\lambda^{\mathrm{c}}(w-\Theta) .
\end{aligned}
$$

The controller can stabilize the saddle steady state of the pendulum if the fixed point $\left(\Theta_{\mathrm{u}}, 0, \Theta_{\mathrm{u}}\right)$ of the controlled system (10) in the extended phase space of variables $(\Theta, \Omega, w)$ is stable. Linearization of the system (10) in the vicinity of the fixed point yields the characteristic equation

$$
\begin{aligned}
\lambda^{3}+ & \left(\beta-\lambda^{\mathrm{c}}\right) \lambda^{2}+\left[k-\left(1-\gamma^{2}\right)^{1 / 2}-\lambda^{\mathrm{c}} \beta\right] \lambda \\
+ & \lambda^{\mathrm{c}}\left(1-\gamma^{2}\right)^{1 / 2}=0 .
\end{aligned}
$$
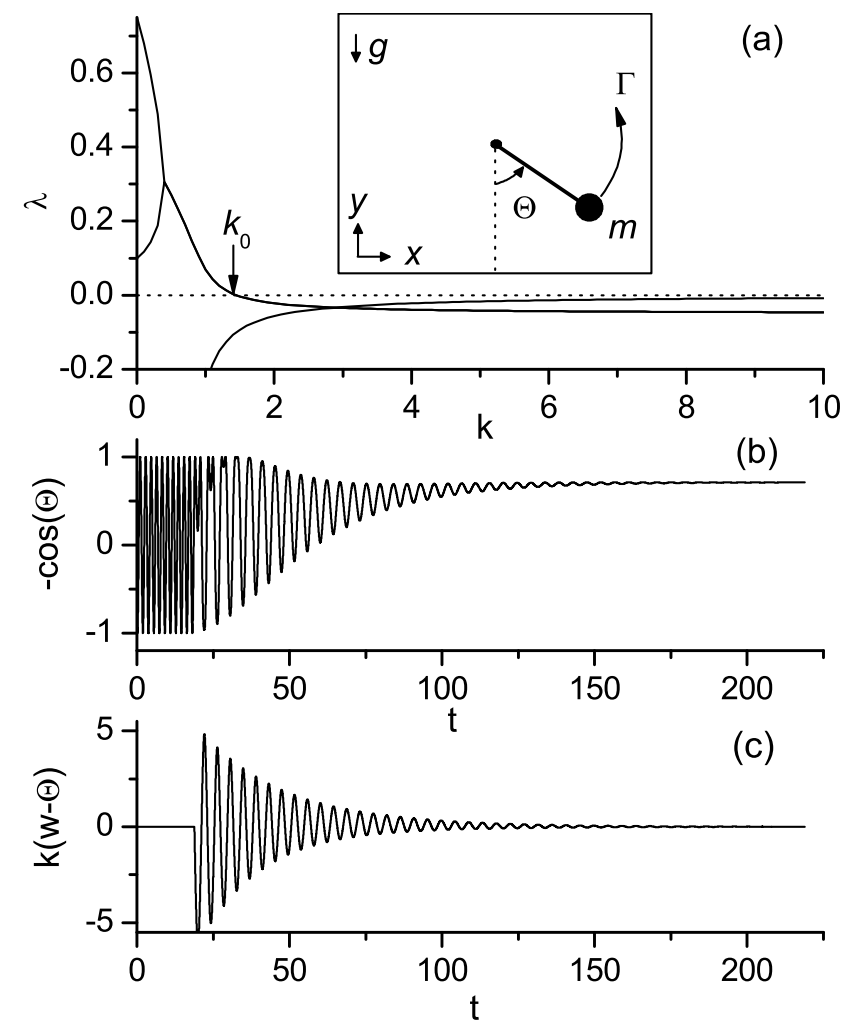

Fig. 2. Stabilization of a saddle steady state of a pendulum driven by a constant torque. (a) Eigenvalues of the controlled pendulum as functions of the control gain $k$. (b) Dynamics of the $y$ variable of the pendulum. (c) Dynamics of the perturbation. The feedback perturbation switching moment is $t=t_{\mathrm{c}}=19$ and the control gain is $k=2.6$.

The fixed point is linearly stable if all the roots of Eq. (11) have negative real parts. Using the well-known Routh-Hurwitz criteria [12] one obtains the following stability conditions:

$$
k>k_{0} \equiv \beta\left(\lambda^{\mathrm{c}}+\frac{\left(1-\gamma^{2}\right)^{1 / 2}}{\beta-\lambda^{\mathrm{c}}}\right), \quad 0<\lambda^{\mathrm{c}}<\beta .
$$

As expected from the above general theory, the necessary stability condition is $\lambda^{\mathrm{c}}>0$, i. e. the saddle steady state can be stabilized only with an unstable controller.

Figure 2(a) shows the real parts of eigenvalues $\lambda$ as functions of the control gain $k$ (calculated at the following set of parameters: $\beta=0.2, \gamma=0.7$, $\lambda^{\mathrm{c}}=0.1$ ). Similarly to the above simple example, the real positive eigenvalues of the pendulum and controller collide on the real axis, pass to the complex plane, and enter the left half-plane at $k=k_{0}$. Results of direct numerical integration of the nonlinear system (10) are presented in Figs. 2(b) and (c). The initial condition of the controller at the switching mo- 
ment $t=t_{\mathrm{c}}=19$ of the control coincides with the current value of the angle, $w\left(t_{\mathrm{c}}\right)=\Theta\left(t_{\mathrm{c}}\right)$. The parameters are chosen in such a way that the uncontrolled pendulum is in a multistable regime: depending on initial conditions it can settle into either a rotating solution where it whirls over the top, or to a stable rest state $\Theta_{\mathrm{s}}$ where the gravity is balanced by the applied torque. For the same values of parameters, there is a coexisting unstable rest state $\Theta_{u}$ of a saddle type. Figure 2(b) shows how the rotating pendulum reaches this state after applying the feedback perturbation. As seen from Fig. 2(c) the perturbation vanishes whenever the stabilization of the saddle steady state is attained. Note that our controller is reference-free, i. e. it does not utilize a knowledge of the position of the fixed point. Thus, it can be used for a tracking procedure. The controlled system will remain in the stabilized saddle state even under a slow variation of the applied torque.

\section{Control of the Lorenz system}

Now we consider the control of stability of the steady states in a chaotic system described by the Lorenz equations [13]

$$
\begin{aligned}
& \dot{x}=\sigma(y-x), \\
& \dot{y}=r x-y-x z, \\
& \dot{z}=x y-b z,
\end{aligned}
$$

where $\sigma, b$, and $r$ are dimensionless parameters. Originally the model (13) has been derived and analysed in the context of turbulent convection. Fortunately, there is a simple physical realization of the Lorenz model: convection in a vertical loop (see inset in Fig. 3) $[14,15]$. The fluid is heated from below, and for strong enough heating convection sets in. Just before the onset, the motion is steady, with a constant velocity $V$. Clearly, due to symmetry, motions in both the clockwise and counter-clockwise directions are possible. If the heating increases, the steady rotation becomes unstable and chaotic reversions of the flow are observed. In the context of the above experiment, variables of the Lorenz equations have the following physical meaning: $x$ is proportional to the flow velocity $V, y$ is proportional to the horizontal temperature difference $T_{3}-T_{1}$, and $z$ is proportional to the vertical temperature difference $T_{4}-T_{2}$. When analysing the Lorenz system the parameters $\sigma$ and $b$ are usually fixed to the values 10 and $8 / 3$, respectively. The

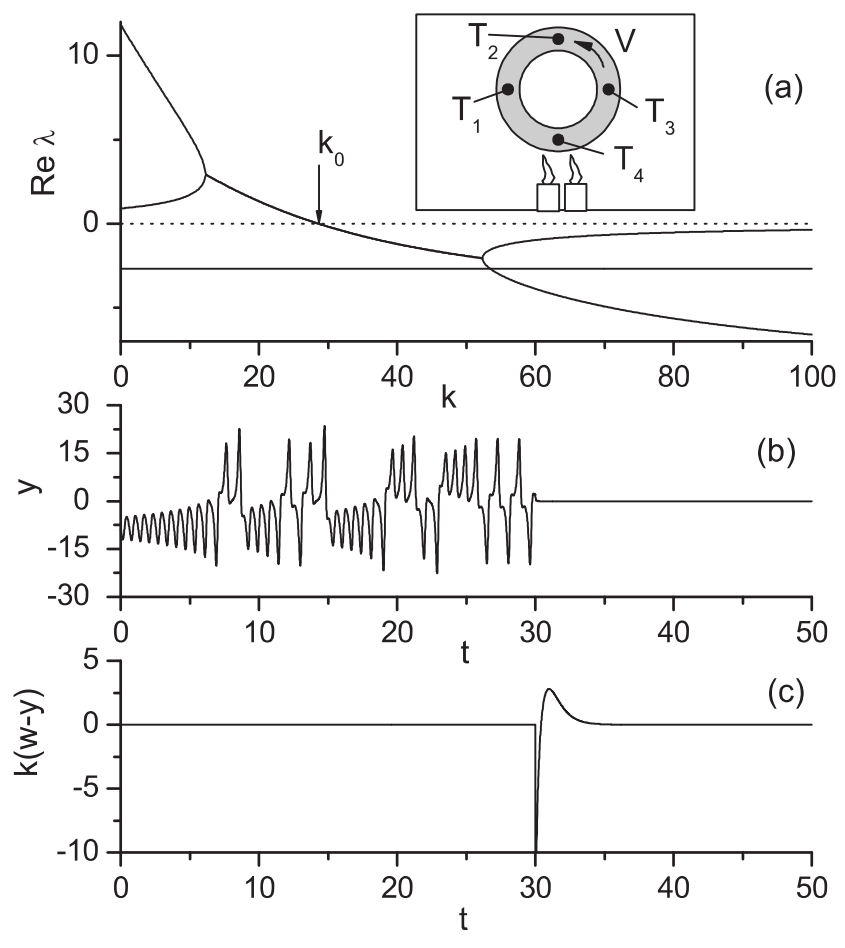

Fig. 3. Stabilization of a saddle steady (nonconvective) state of the Lorenz system with an unstable controller. (a) Eigenvalues of the controlled Lorenz system as functions of the control gain $k$. (b) Dynamics of the $y$ variable. (c) Dynamics of the perturbation. The set of parameters used is $\sigma=10, b=8 / 3, r=28$, $\lambda^{\mathrm{c}}=1$. The feedback perturbation is switched on at the moment $t=t_{\mathrm{c}}=30$. The initial condition of the controller at this moment coincides with the observable $y, w\left(t_{\mathrm{c}}\right)=y\left(t_{\mathrm{c}}\right)$. The control gain is $k=55$.

parameter $r$ is proportional to the heating rate at the bottom and is usually taken as a main control parameter.

For $0<r<1$, the Lorenz system has a unique stable steady state, a stable node, at the origin $(x, y, z)=$ $(0,0,0)$. For $r>1$, the origin becomes a saddle. This means that the motionless state of the liquid looses stability. Just at this bifurcation, two additional symmetrical stable steady states $\left([b(r-1)]^{1 / 2},[b(r-1)]^{1 / 2}, r-1\right)$ and $\left(-[b(r-1)]^{1 / 2},-[b(r-1)]^{1 / 2}, r-1\right)$ appear. They correspond to the stationary motion of the liquid with the constant velocity. For $r>r_{h}=\sigma(\sigma+b+3) /$ $(\sigma-b-1)$, these fixed points loose their stability and become unstable spirals. Now a chaotic motion of the liquid is observed in the system. Our aim is to stabilize all unstable steady states of the system in a chaotic regime when $r>r_{h}$, using the above adaptive dynamic controller.

We suppose that an observable is $y$ and we can influence the temperature difference $T_{3}-T_{1}$ by additional heating of the loop in the horizontal direction. Then we 
analyse the controlled Lorenz system described by the following equations:

$$
\begin{aligned}
& \dot{x}=\sigma(y-x), \\
& \dot{y}=r x-y-x z+k(w-y), \\
& \dot{z}=x y-b z, \\
& \dot{w}=\lambda^{\mathrm{c}}(w-y) .
\end{aligned}
$$

We start with the problem of stabilizing the motionless steady state, i. e. the saddle fixed point at the origin. This fixed point has two negative and one positive real eigenvalues. Thus, it can be stabilized only with an unstable controller with the positive parameter $\lambda_{\mathrm{c}}$. Indeed, linearizing the system (14) in the vicinity of the origin $(x, y, z, w)=(0,0,0,0)$ we obtain that one eigenvalue is independent of $k$ and is negative, $\lambda=-b$, while remaining three eigenvalues satisfy the characteristic equation

$$
\begin{aligned}
\lambda^{3} & +\left(\sigma+1-\lambda^{\mathrm{c}}+k\right) \lambda^{2} \\
& +\left[\sigma\left(1-r-\lambda^{\mathrm{c}}+k\right)-\lambda^{\mathrm{c}}\right] \lambda \\
& +\sigma \lambda^{\mathrm{c}}(r-1)=0 .
\end{aligned}
$$

The necessary condition of stability of the polynomial (15) is $\sigma \lambda^{\mathrm{c}}(r-1)>0$. The latter can be satisfied only with an unstable controller, $\lambda^{\mathrm{c}}>0$. Using the Routh-Hurwitz criteria [12] one can obtain the threshold of the stability $k_{0}$. For $\sigma=10, r=$ 28 , and sufficiently small $\lambda^{\mathrm{c}}$, the stability condition reads

$$
k>k_{0}\left(\lambda^{\mathrm{c}}\right) \approx 27+1.81 \lambda^{\mathrm{c}} .
$$

Figure 3(a) presents the three largest real parts of the eigenvalues as functions of the control gain $k$. The horizontal line in the figure corresponds to the eigenvalue $\lambda=-b$ that is independent of $k$. Results of direct integration of the nonlinear system (14) are presented in Figs. 3(b) and (c). Initially the system is in a chaotic regime. After the perturbation was switched on, the system is forced to the rest state. Whenever the stabilization of the rest state is attained the feedback perturbation vanishes.

Similar results of adaptive stabilization of the unstable spirals $\left( \pm[b(r-1)]^{1 / 2}, \pm[b(r-1)]^{1 / 2}, r-1\right)$ are shown in Fig. 4. These fixed points correspond to the stationary motion of the liquid in clockwise and counter-clockwise directions with a constant ve-

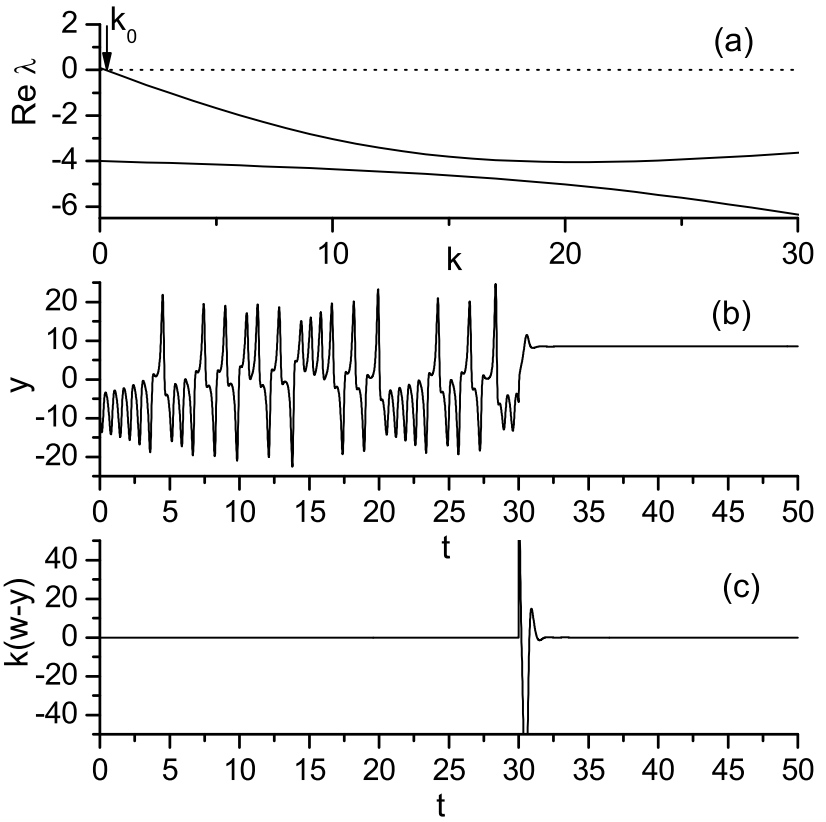

Fig. 4. Stabilization of an unstable spiral (the convective state with a constant velocity of the fluid) of the Lorenz system with a stable controller. The values of parameters are the same as in Fig. 3, except $\lambda^{\mathrm{c}}$ and $k$ that here are equal to -4 and 20, respectively.

locity. They have identical stability conditions and depending on initial conditions the system can be stabilized to either of these states. The unstable spirals have one real negative eigenvalue and a complex conjugate pair of eigenvalues with the positive real parts. Thus, we use a stable controller for the stabilization. Physically, this stabilization means that chaotic convection of the system is transformed into regular convection with a constant velocity of the liquid. We emphasize that this state can be maintained just by applying tiny feedback perturbation.

\section{Control of an electrochemical oscillator}

Lastly, we demonstrate the use of adaptive controller with control in an electrodissolution process, the dissolution of nickel in sulphuric acid. The main features of the process can be qualitatively described with a model proposed by Haim et al. [16]. The dimensionless model reads

$$
\begin{aligned}
\dot{e} & =\frac{V-e}{R}-f_{3}(e)(1-\Theta), \\
\Gamma_{1} \dot{\Theta} & =f_{1}(e)(1-\Theta)-f_{2}(e) \Theta .
\end{aligned}
$$


Here $e$ is the dimensionless potential of the $\mathrm{Ni}$ electrode and $\Theta$ is the surface coverage of $\mathrm{NiO}+\mathrm{NiOH}$. The functions in the above expressions are

$$
\begin{aligned}
& f_{1}(e)=\frac{\exp (0.5 e)}{1+C_{h} \exp (e)}, \\
& f_{2}(e)=\frac{b C_{h} \exp (2 e)}{C_{h} C+\exp (e)}, \\
& f_{3}(e)=C_{h} f_{1}(e)+a \exp (e) .
\end{aligned}
$$

An observable is the current through the Ni electrode

$$
i=\frac{V-e}{R},
$$

where $R$ is the series resistance of the cell and $V$ is the circuit potential, the main experimentally controlled parameter. We shall control the system dynamics by varying this parameter, $V=V_{0}+\delta V$, with the feedback signal $\delta V$ derived from the observable $i(t)$. But first we consider the steady-state solutions and dynamical properties of the free system, when $\delta V=0$, i. e. $V=V_{0}=$ const.

\subsection{Analysis of the free system}

For a fixed voltage $V=V_{0}$, the steady-state solutions $\left(e_{0}, \Theta_{0}\right)$ of the system (17) are determined by the equations

$$
\begin{gathered}
\frac{V_{0}-e_{0}}{R}-f_{3}\left(e_{0}\right)\left(1-\Theta_{0}\right)=0, \\
f_{1}\left(e_{0}\right)\left(1-\Theta_{0}\right)-f_{2}\left(e_{0}\right) \Theta_{0}=0 .
\end{gathered}
$$

The equations are linear with respect to variables $V_{0}$ and $\Theta_{0}$. Thus, we can easily obtain explicit expressions

$$
\begin{aligned}
& \Theta_{0}=\Theta_{0}\left(e_{0}\right)=\frac{f_{1}\left(e_{0}\right)}{f_{1}\left(e_{0}\right)+f_{2}\left(e_{0}\right)}, \\
& V_{0}=V_{0}\left(e_{0}\right)=e_{0}+R \frac{f_{2}\left(e_{0}\right) f_{3}\left(e_{0}\right)}{f_{1}\left(e_{0}\right)+f_{2}\left(e_{0}\right)},
\end{aligned}
$$

that define the steady-state characteristics of the system in a parametric form ( $e_{0}$ is interpreted as an independent parameter). The steady-state characteristics $e_{0}$ versus $V_{0}$ and $i$ versus $V_{0}$ are shown in Fig. 5. We see that in a certain interval of the potential $V_{0}$ the system has three coexisting fixed points. The linear analysis of these points shows that the lower branch in Fig. 5(a) corresponds to an unstable spiral, the middle branch represents a saddle, and the upper branch is a stable node.
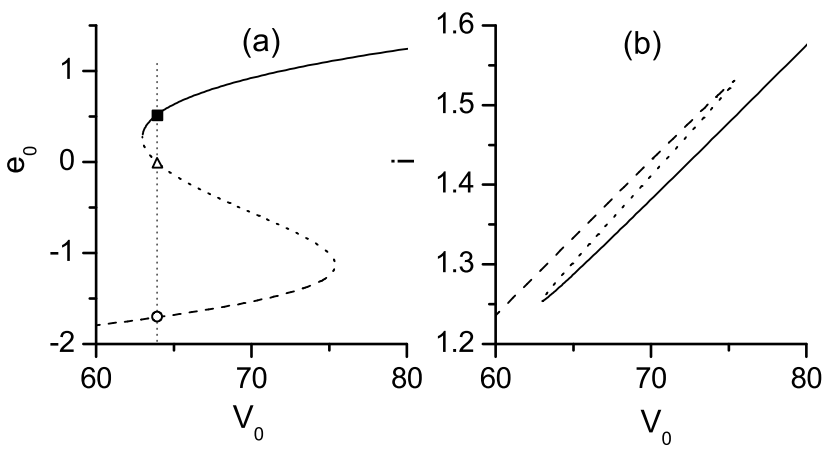

Fig. 5. Steady-state characteristics of the chemical reaction obtained from Eqs. (20). The dashed line represents an unstable spiral, the dotted line corresponds to a saddle, and the solid one is a stable node. The values of parameters are $R=50, \Gamma_{1}=10^{-2}$, $b=6 \cdot 10^{-5}, C_{h}=1600, C=10^{-3}, a=0.3$.

Now we discuss the bifurcations that appear in the system when varying the control parameter $V_{0}$. The phase portraits of the system for different values of $V_{0}$ are shown in Fig. 6. The results are presented in the delayed phase space coordinates $(i(t), i(t-\tau))$ using the observable $i(t)$. Such a choice of the phase space variables allows us to compare the numerical results with the experimental ones.

For small values $V_{0}$, an unstable spiral is the only steady state of the system. The spiral is surrounded by a stable limit cycle that corresponds to chemical oscillations (Fig. 6(a)). The increase of $V_{0}$ leads to a saddle-node bifurcation at which two additional fixed points appear (Fig. 6(b)). Then the saddle collides with the limit cycle (homoclinic bifurcation, Fig. 6(c)) and the limit cycle disappears (Fig. 6(d)). The further increase of $V_{0}$ leads to another saddle-node bifurcation (Fig. 6(e)) and the only one fixed point, a stable node, remains in the system (Fig. 6(f)).

Next, we fix the value of the voltage $V_{0}=63.888$ (which is marked in Fig. 5(a) by a vertical dotted line). Then the system has two unstable fixed points: the saddle and the unstable spiral, the coordinates $\left(e_{0}, \Theta_{0}\right)$ of which are $(0.0,0.0166)$ and $(-1.7074,0.4521)$, respectively. We consider the problem of stabilizing these unstable states by using two different strategies. In the first, the value of the potential $e$ is taken as a control signal. We suppose that this value can be reconstructed from the observable $i$. In the second, we design the controller that uses directly the observable $i$.

\subsection{Stabilization using the potential as a control signal}

Since the input variable, the voltage $V$, perturbs only the first differential equation of the system (17), it is 

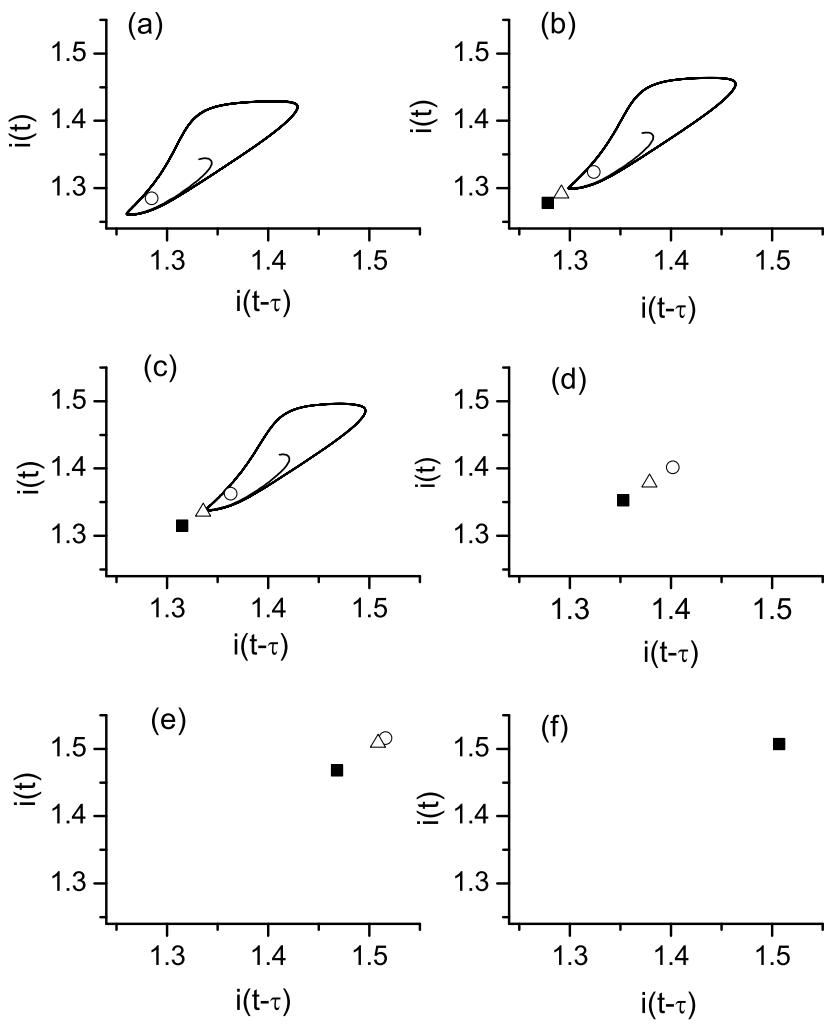

Fig. 6. Phase portraits of the free chemical system presented in delayed coordinates $(\tau=0.94)$ for different values of the voltage $V_{0}$ : (a) 62.5, (b) 64.5, (c) 66.5, (d) 68.5, (e) 74.5, and (f) 76.5. The circles, triangles, and squares denote unstable spirals, saddles, and stable nodes, respectively. The values of parameters are the same as in Fig. 5.

natural to construct the perturbation in such a way that it introduces a negative feedback to the potential $e$. If the value of the circuit resistance $R$ is known then the value of the potential $e$ can be reconstructed from the observable $i$ and the input variable $V, e=V-i R$. However, the exact value $R$ is unknown in experiment. Then we propose to use the difference $\widetilde{e}=V-i r$ as an effective control signal, where $r$ is an adjustable control parameter which is not required to be exactly equal to the circuit resistance $R$. We shall see that the stabilization can be attained for $r \neq R$ as well.

Here we restrict ourselves to the problem of stabilizing the saddle steady state that requires a use of an unstable controller. Introducing an effective potential $\widetilde{e}$ we define an unstable controller by the differential equation

$$
\frac{\mathrm{d} w}{\mathrm{~d} t}=\lambda^{\mathrm{c}}(w-\widetilde{e})=\lambda^{\mathrm{c}}(w-V+i r),
$$

where $w$ is a dynamic variable of the controller and $\lambda^{\mathrm{c}}$ is a positive constant. Now we feed back the control perturbation $\delta V=k(w-V+i r)$ to the adjustable parameter $V$,

$$
V=V_{0}+k(w+i r-V) .
$$

Solving this equation together with Eq. (19) we obtain the following expression for the voltage:

$$
V=\frac{V_{0}+k(w-e / R)}{1+k(1-r / R)}
$$

where $k$ is the control gain. Thus, the controlled chemical system is defined by differential Eqs. (17) and (22) and algebraic expressions (18), (19), and (24). Note that the introduced controller does not change the steady-state solutions of the free chemical system. Indeed, the stationary state of the controller is determined by the equality $w-V+i r=0$. Thus, the perturbation added to the voltage is equal to zero and from Eq. (23) we obtain that $V=V_{0}$. The steady-state value of the controller variable is $w_{0}=V_{0}(1-r / R)+e_{0} r / R$ and the corresponding fixed point of the controlled chemical system in the whole phase space of variables $(e, \Theta, w)$ is $\left(e_{0}, \Theta_{0}, w_{0}\right)$, where $\left(e_{0}, \Theta_{0}\right)$ are the steady-state solutions of the free chemical system. Linearizing Eqs. (17) and (22) in the vicinity of this fixed point one can obtain the stability conditions. Figure 7(a) shows the eigenvalues of the saddle fixed point $\left(e_{0}, \Theta_{0}, w_{0}\right)=(0.0,0.1666,-12.7776)$, taken at the voltage $V_{0}=63.888$, as functions of the control gain $k$. For $k>k_{0} \approx 2.97$, the saddle steady state of the free systems becomes stable due to introduced feedback control. This result of a linear theory is confirmed by direct integration of nonlinear Eqs. (17) and (22). Figures 7(c) and (d) show the dynamics of the current and perturbation, respectively, for $k=0.02$. At the moment of switching on the control $t=t_{\mathrm{c}} \approx 93$ the initial condition for the controller is $w\left(t_{\mathrm{c}}\right)=\widetilde{e}\left(t_{\mathrm{c}}\right)$. Note that the parameter $r$ is chosen not equal to $R$ ( $r=60$ and $R=50$ ). Although the effective potential $\widetilde{e}$ does not coincide with the real potential $e$, the stabilization of the fixed point is still possible.

\subsection{Stabilization using the current as a control signal}

The controller described in previous paragraph is based on reconstruction of the potential $e$ and implementation of a negative feedback to this variable. Now we consider another controller that does not require any reconstructions of dynamic variables and is particularly convenient for experimental implementation. We take the observable $i(t)$ as a control signal, transform it by 


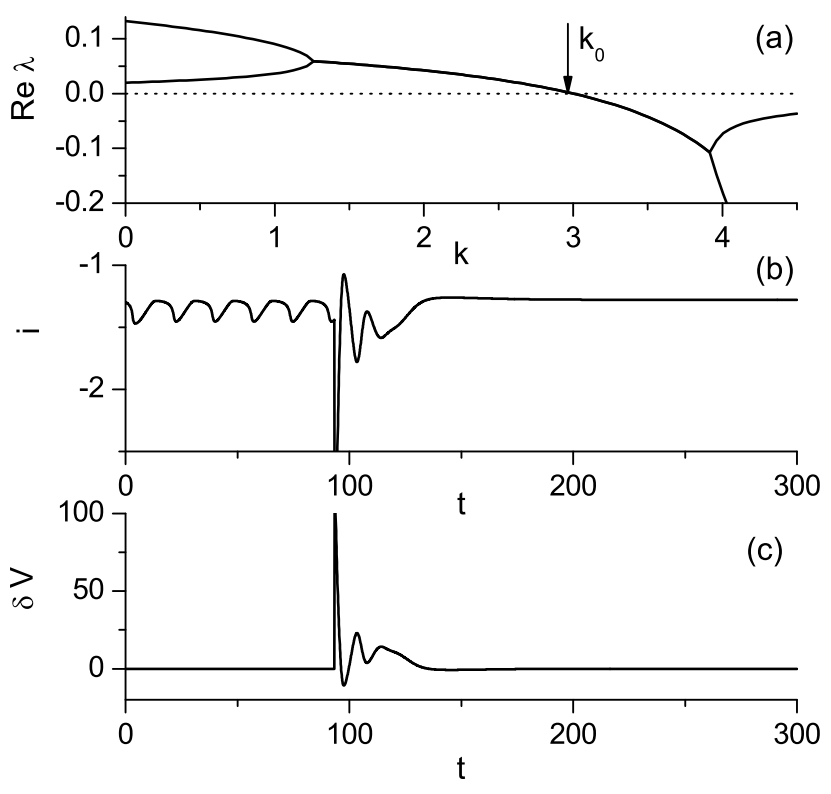

Fig. 7. Stabilization of a saddle steady state of the chemical system using the potential as a control signal. (a) Eigenvalues of the controlled chemical system as functions of the control gain $k$. (b, c) Dynamics of the current and perturbation, respectively. The parameters of the controller are $\lambda^{\mathrm{c}}=0.02, r=60, k=3.9$. The feedback perturbation is switched on at the moment $t=t_{\mathrm{c}}=93$.

an adaptive dynamic controller, and feed back the output to the control parameter $V(t)$. Specifically, we define an adaptive controller by the differential equation

$$
\frac{\mathrm{d} w}{\mathrm{~d} t}=\lambda^{\mathrm{c}}(w-i)
$$

where $w$ is a dynamic variable of the controller and $\lambda^{\mathrm{c}}$ is a characteristic parameter. Then we feed back the control perturbation $\delta V=k(i-w)$ to the input voltage $V$,

$$
V=V_{0}+\delta V=V_{0}+k(i-w),
$$

where $k$ is the control gain. Substituting this $V$ expression into Eq. (19) and solving it with respect to unknown current, we obtain $i=\left(V_{0}-e-k w\right) /(R-k)$. Then from Eq. (26) we obtain the following expression for the voltage:

$$
V=V_{0}+k \frac{V_{0}-e-w R}{R-k} .
$$

We see that the voltage perturbation is singular for $k=R$. The controlled chemical system is defined by differential Eqs. (17) and (25) and algebraic expressions (18), (19), and (27). This controller as well as considered before does not change the steady state solutions of the free chemical system. Whenever the controller attains the steady state, $\mathrm{d} w / \mathrm{d} t=0$, the voltage perturbation $\delta V=k(i-w)$ vanishes. The steady-state value of the controller variable coincides with the steady-state value of the current of the unperturbed system, $w_{0}=i_{0}=\left(V_{0}-e_{0}\right) / R$. Thus, the fixed point of the controlled chemical system in the whole phase space of variables $(e, \Theta, w)$ is $\left(e_{0}, \Theta_{0}, i_{0}\right)$. Linearizing Eqs. (17) and (25) in the vicinity of this fixed point one can obtain the dependence of its eigenvalues on the control gain $k$. For the unstable spiral $\left(e_{0}, \Theta_{0}, i_{0}\right)=(-1.7074,0.4521,1.3119)$, taken at the voltage $V_{0}=63.888$, this dependence is shown in Fig. 8(a). For $k>k_{0} \approx 46.2$, the initially unstable spiral becomes stable. Figures 8(b) and (c) show the results of a nonlinear analysis. The dynamics of the current and perturbation are obtained by direct integration of the nonlinear system of Eqs. (17) and (25). At the moment of switching on the control $t=t_{\mathrm{c}}=90$ the initial condition for the controller is taken to be equal to the current, $w\left(t_{\mathrm{c}}\right)=i\left(t_{\mathrm{c}}\right)$. Again, the current of the controlled chemical system asymptotically converges to the unperturbed steadystate value $i_{0}$ and the perturbation vanishes. To stabilize the unstable spiral we used a stable controller with the negative parameter $\lambda^{\mathrm{c}}=-0.01$. Figure 9 shows similar results of stabilizing the saddle point $\left(e_{0}, \Theta_{0}, i_{0}\right)=(0.0,0.0166,1.27778)$ taken at the same voltage $V_{0}=63.888$. However, now an unstable controller with the positive parameter $\lambda^{\mathrm{c}}=0.01$ has been used.

Note that the controller considered in this section has only one adjustable parameter $\lambda^{\mathrm{c}}$, while the controller based on reconstruction of the potential $e$ has two adjustable parameters, $\lambda^{\mathrm{c}}$ and $r$. Thus, this controller is more convenient for experimental implementation.

\section{Restriction of perturbation and basins of attraction}

The linear stability of a fixed point of dynamical system guarantees its stabilization only for the initial conditions that are close to the fixed point. Important questions arise what is the basin of attraction of a linear stable fixed point in the phase space of nonlinear system and how to control the size of this basin? These questions are especially significant from an experimental point of view. Generally, there is no analytical approach to answer these questions. The basins of attraction can be obtained numerically by direct integration of the underlying differential equations for different values of initial conditions. We performed such an analysis for the chemical system in the case when 

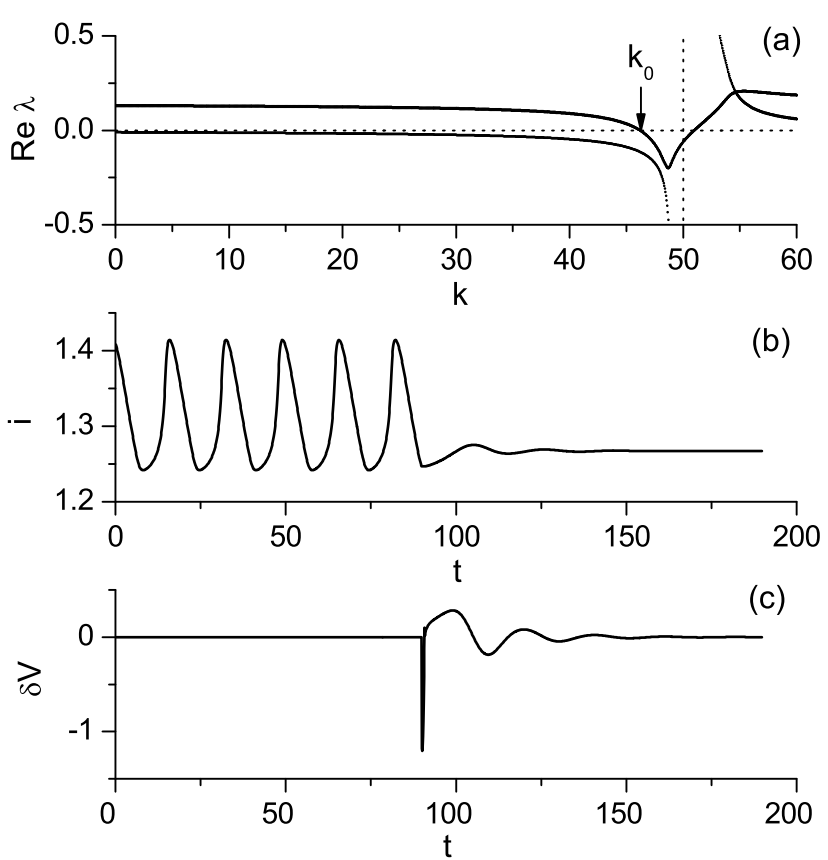

Fig. 8. Stabilization an unstable spiral of the chemical system using the current as a control signal. (a) Eigenvalues of the controlled chemical system as functions of the control gain $k$. (b, c) Dynamics of the current and perturbation, respectively. The parameters of the controller are $\lambda^{\mathrm{c}}=-0.01$ and $k=49.9$. The feedback perturbation is switched on at the moment $t=t_{\mathrm{c}}=90$.

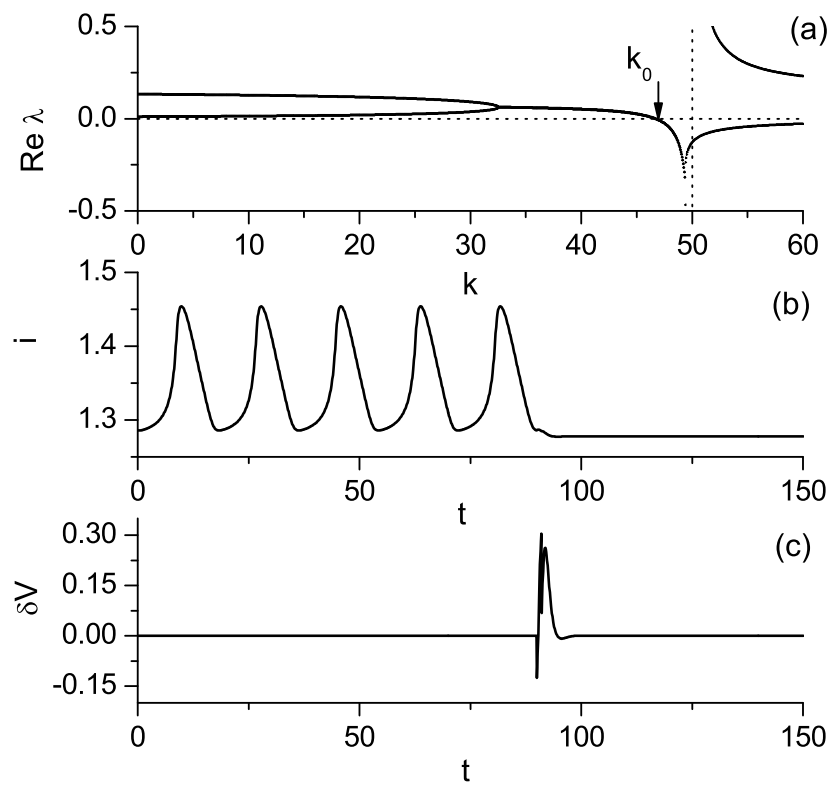

Fig. 9. Stabilization of an unstable spiral of the chemical system using the current as a control signal. (a) Eigenvalues of the controlled chemical system as functions of the control gain $k$. (b,c) Dynamics of the current and perturbation, respectively. The parameters of the controller are $\lambda^{\mathrm{c}}=0.01$ and $k=49.9$. The feedback perturbation is switched on at the moment $t=t_{\mathrm{c}}=90$.
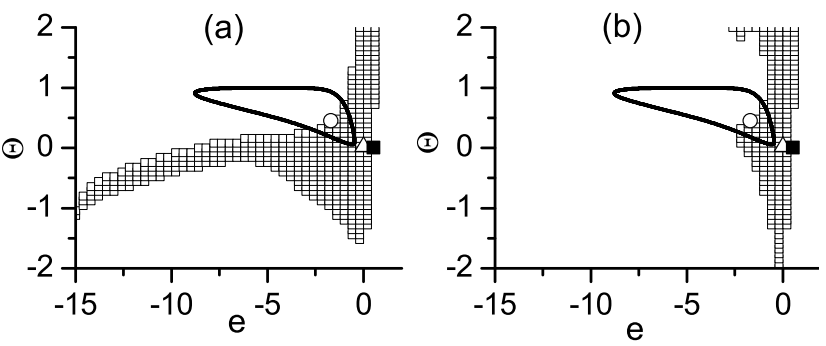

(c)

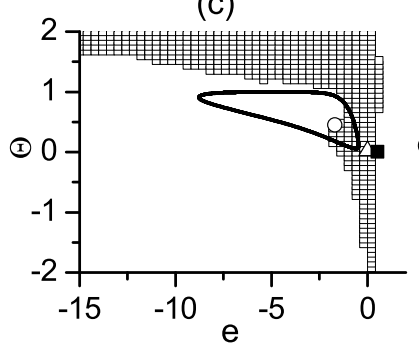

(d)

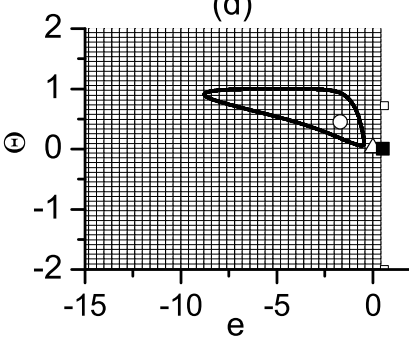

Fig. 10. Basins of attraction of the stabilized saddle steady state of the chemical system without restriction of the perturbation (a) and for restricted perturbation with different values of $\delta V_{\max }$ : (b) 100 , (c) 50, and (d) 25. The solid line, as well as in Fig. 6, represents the limit cycle. The circles, triangles, and squares denote spirals, saddles, and nodes, respectively.

the current is used as a control signal. The basin of attraction in the $(e, \Theta)$ phase plane for the stabilized saddle fixed point is presented in Fig. 10(a). The initial condition for the controller is taken to be equal to the initial value of the current, $w(0)=i(0)=$ $\left(V_{0}-e(0)\right) / R$. We see that only a small part of the limit cycle resides in the basin of attraction of the saddle point. It means that the control will not be successful always if we try to switch the system behaviour from a regime of limit cycle oscillations to a saddle steady state. We will succeed in our intention only if we switch on a control at a proper moment when the phase of limit cycle oscillations is in the basin of attraction of the saddle steady state. If the control is switched on at an improper moment, the feedback perturbation grows up rapidly and the system runs far away from the saddle point and the limit cycle. A natural way to avoid the runaways is to restrict the perturbation.

We analysed the system behaviour under the following algorithm of restriction. When the absolute value of the perturbation $|\delta V|$ reaches some maximum $\delta V_{\max }$ we zero the perturbation by changing the state of the controller. Let $t_{m}$ be the moment when the perturbation reaches the maximum of the allowable amplitude, $\left|\delta V\left(t_{m}\right)\right|=\delta V_{\max }$. At this moment we change the state of the controller by setting the variable $w$ to be equal to the current, $w\left(t_{m}\right)=i\left(t_{m}\right)$. From Eq. (26) 
it follows that the perturbation at this moment drops to zero, $\delta V\left(t_{m}\right)=0$. Figures $10(\mathrm{~b})$, (c), and (d) show the evolution of the basin of attraction when varying the parameter $\delta V_{\max }$. For sufficiently small $\delta V_{\max }$ the basin of attraction becomes rather large so that the limit cycle totally resides within the basin of attraction (Fig. 10(d)). In this case the system behaviour can be changed from periodic limit cycle oscillations to a saddle steady state by switching on the control at any moment independent of the phase of oscillations. Thus, the constrained perturbations can essentially improve the control of dynamical system in a real experiment.

In a similar manner, we analysed the basin of attraction for the spiral fixed point. The analysis shows that this basin is rather large (similar to that presented in Fig. 10(d)) even without restriction of the perturbation. The different sizes of attraction basins for the spiral and saddle fixed points are probably related to the fact that different controllers have been used for the stabilization. For the saddle fixed point we used the unstable controller, while the spiral is stabilized with the stable one. The unstable controller increases the probability of runaway and to avoid it a restriction of the perturbation is required. The stable controller does not introduce any additional instabilities to the system and the restriction on the perturbation is not so essential.

\section{Conclusions}

In the present paper we have considered an adaptive dynamic controller for stabilizing unknown unstable steady states of dynamical systems. The controller automatically finds the position of the unstable fixed point in the phase space and stabilizes it. Whenever the stabilization is attained the feedback perturbation vanishes and there is no power dissipation in the feedback loop. The simplest example of such a controller is a conventional low-pass filter. However, it has a topological limitation similar to that of a timedelayed feedback controller. The controller cannot stabilize unstable steady states with an odd number of real positive eigenvalues. We prove this limitation in a rather general way. To avoid the limitation we introduce an unstable degree of freedom into a controller and show that such an unstable controller is capable to stabilize the saddle steady states of dynamical systems.
The efficiency of the adaptive controller is demonstrated for several models: a pendulum driven with a constant torque, the Lorenz system, and an electrochemical oscillator. We analyse the basin of attraction of the stabilized fixed point in the phase space and show that this basin can be essentially enlarged by a restriction of the feedback perturbation. A recent experiment [17] with the electrochemical $\mathrm{Ni}$ dissolution system confirms the theoretical results presented in this paper.

\section{References}

[1] R. Bellman, Introduction to the Mathematical Theory of Control Processes (Academic Press, New York, 1971).

[2] G. Stephanopoulos, Chemical Process Control: An Introduction to Theory and Practice (Prentice Hall, Englewood Cliffs, NJ, 1984).

[3] E. Ott, C. Grebogi, and J.A. Yorke, Controlling chaos, Phys. Rev. Lett. 64, 1196-1199 (1990).

[4] Handbook of Chaos Control, ed. H.G. Shuster (WileyVCH, Weiheim, 1999).

[5] K. Pyragas, Continuous control of chaos by selfcontrolling feedback, Phys. Lett. A 170, 421-428 (1992).

[6] H. Nakajima, On analytical properties of delayed feedback control of chaos, Phys. Lett. A 232, 207-210 (1997).

[7] K. Pyragas, Control of chaos via an unstable delayed feedback controller, Phys. Rev. Lett. 86, 2265-2268 (2001).

[8] K. Pyragas, Control of chaos via extended delay feedback, Phys. Lett. A 206, 323-330 (1995).

[9] A. Namajūnas, K. Pyragas, and A. Tamaševičius, Stabilization of an unstable steady-state in a MackeyGlass system, Phys. Lett. A 204, 255-262 (1995).

[10] N.F. Rulkov, L.S. Tsimring, and H.D.I. Abarbanel, Tracking unstable orbits in chaos using dissipative feedback control, Phys. Rev. E 50, 314-324 (1994).

[11] A.S.Z. Schweinsberg and U. Dressler, Characterization and stabilization of the unstable fixed points of a frequency doubled Nd: YAG laser, Phys. Rev. E 63, 056210 (2001).

[12] K. Ogata, Modern Control Engineering (Prentice Hall, Englewood Cliffs, NJ, 1990).

[13] E.N. Lorenz, Deterministic nonperiodic flow, J. Atmos. Sci. 20, 130-141 (1963).

[14] M. Gorman, P.J. Widmann, and K.A. Robbins, Chaotic flow regimes in a convective loop, Phys. Rev. Lett. 52(25), 2241-2244 (1984).

[15] M. Gorman, P.J. Widmann, and K.A. Robbins, Nonlinear dynamics of a convection loop: A quantitative comparison of experiment with theory, Physica D 19(2), 255-267 (1986). 
[16] D. Haim, O. Lev, L.M. Pismen, and M. Sheintuch, Modelling periodic and chaotic dynamics in anodic nickel dissolution, J. Phys. Chem. 96, 2676-2681 (1992).
[17] K. Pyragas, V. Pyragas, I.Z. Kiss, and J.L. Hudson, Stabilizing and tracking unknown steady states of dynamical systems, Phys. Rev. Lett. 89, 244103 (2002).

\title{
NETIESINIŲ DINAMINIU SISTEMŲ NESTABILIŲJU RIMTIES BŪSENŲ ADAPTYVUSIS STABILIZAVIMAS
}

\author{
V. Pyragas, K. Pyragas \\ Puslaidininkiu fizikos institutas, Vilnius, Lietuva
}

\section{Santrauka}

Nagrinètas dinaminis grižtamojo ryšio valdiklis nežinomoms dinaminiu sistemų rimties būsenoms stabilizuoti. Irodyta, kad rimties būsena negali būti stabilizuota, jeigu sistema ir valdiklis kartu turi nelygini realiujų teigiamų tikrinių verčių skaičių. Antros eilès sistemoms toks topologinis ribojimas reiškia, kad stabiliuoju valdikliu galima stabilizuoti tik nestabiliają spiralę arba nestabilụji mazgą, o balnui stabilizuoti grižtamojo ryšio kilpoje reikalingas papildomas nestabilusis laisvès laipsnis. Valdiklio naudingumas balnams bei nestabiliosioms spiralems stabilizuoti yra parodytas skaitiškai keliems modeliams: svyruoklei, veikiamai jẻgos momento, Lorenz'o sistemai ir elektrocheminei Ni tirpimo reakcijai. 